\title{
Large holes in quasi-random graphs
}

\author{
Joanna Polcyn \\ Department of Discrete Mathematics \\ Adam Mickiewicz University \\ Poznań, Poland \\ joaska@amu.edu.pl
}

Submitted: Nov 23, 2006; Accepted: Apr 10, 2008; Published: Apr 18, 2008

\begin{abstract}
Quasi-random graphs have the property that the densities of almost all pairs of large subsets of vertices are similar, and therefore we cannot expect too large empty or complete bipartite induced subgraphs in these graphs. In this paper we answer the question what is the largest possible size of such subgraphs. As an application, a degree condition that guarantees the connection by short paths in quasi-random pairs is stated.
\end{abstract}

\section{Introduction}

Szemerédi's Regularity Lemma for graphs [6] has become one of the most important tools in the modern graph theory. When solving certain problems, this Lemma allows us to concentrate on quasi-random subgraphs (called also $\varepsilon$-regular pairs) instead of considering the whole graph. Notable examples of this method can be found in $[2,3]$. This approach is very convenient since such regular pairs have a lot of nice properties. In particular, in quasi-random graphs the densities of almost all pairs of large subsets of vertices are similar, and therefore we cannot expect too large empty induced subgraphs (holes) in these graphs.

The problem of holes in $\varepsilon$-regular pairs was already studied in [4]. Let $h(\varepsilon, d, n)$ be defined as the largest integer $h$ such that every balanced bipartite graph $G$ with $2 n$ vertices and density at least $d$, contains a subgraph $H$ on $h+h$ vertices and with no hole with at least $\varepsilon n$ vertices on each side of the bipartition. The authors, having given $0<\varepsilon, d<1$ and a positive integer $n$, estimate the number $h(\varepsilon, d, n)$. In this paper we study a similar problem. With given $d$ and $\varepsilon$ we determine the maximal size of a hole that can be contained in some, sufficiently large, $(d ; \varepsilon)$-regular graph. As a corollary, the size of a largest complete bipartite graph that can be contained in a $(d ; \varepsilon)$-regular pair is also given. 
We start with some preliminary facts and definitions. Let $G=(V, E)$ be a graph with a vertex set $V=V(G)$ and an edge set $E=E(G) \subset[V]^{2}$. For $U, W \subseteq V$ define

$$
\mathrm{e}_{G}(U, W)=|\{(x, y): x \in U, y \in W,\{x, y\} \in E\}| .
$$

Moreover, for nonempty and disjoint $U$ and $W$ let

$$
\mathrm{d}_{G}(U, W)=\frac{\mathrm{e}_{G}(U, W)}{|U||W|}
$$

be the density of the graph $G$ between $U$ and $W$, or simply, the density of the pair $(U, W)$.

In the rest of this paper we assume that $G$ is a bipartite graph with bipartition $V=V_{1} \cup V_{2}$. A standard averaging argument yields the following fact.

Fact 1.1 If $\mathrm{d}_{G}\left(V_{1}, V_{2}\right)<d[>d]$, then for all natural numbers $\ell_{1} \leq\left|V_{1}\right|$ and $\ell_{2} \leq\left|V_{2}\right|$ there exist subsets $U \subset V_{1},|U|=\ell_{1}$ and $W \subset V_{2},|W|=\ell_{2}$ with $\mathrm{d}_{G}(U, W)<d[>d]$.

Definition 1.2 Given $\varepsilon_{1}, \varepsilon_{2}>0$, a bipartite graph $G$ with bipartition $\left(V_{1}, V_{2}\right)$, where $\left|V_{1}\right|=n$ and $\left|V_{2}\right|=m$, is called $\left(\varepsilon_{1}, \varepsilon_{2}\right)$-regular if for each pair of subsets $U \subseteq V_{1}$ and $W \subseteq V_{2},|U| \geq \varepsilon_{1} n,|W| \geq \varepsilon_{1} m$, the inequalities

$$
d-\varepsilon_{2}<\mathrm{d}_{G}(U, W)<d+\varepsilon_{2}
$$

hold for some real number $d>0$. We may then also say that $G$, or the pair $\left(V_{1}, V_{2}\right)$, is $\left(d ; \varepsilon_{1}, \varepsilon_{2}\right)$-regular. Moreover, if $\varepsilon_{1}=\varepsilon_{2}=\varepsilon$, we will use the names $(d ; \varepsilon)$-regular and $\varepsilon$-regular.

For example, according to the above definition, a complete bipartite graph has its density equal to 1 . Therefore it is $\varepsilon$-regular for all $\varepsilon>0$.

Remark 1.3 Each $\left(\varepsilon_{1}, \varepsilon_{2}\right)$-regular graph is $\varepsilon$-regular for all $\varepsilon \geq \max \left\{\varepsilon_{1}, \varepsilon_{2}\right\}$. Note also that checking if the given graph is $\left(d ; \varepsilon_{1}, \varepsilon_{2}\right)$-regular we need to consider only sets of the size $\varepsilon_{1}\left|V_{i}\right|, i=1,2$.

In the following section we state our main results proved in Sections 3. In Section 4, as an applications, we present a degree condition that guarantees the connection by short paths in quasi-random pairs.

\section{Main results}

From the definition of a $(d ; \varepsilon)$-regular pair it follows that the densities of most pairs of subsets of vertices are close to $d$. However, it turns out that even in such highly regular graphs, some pairs of small subsets may have their densities far from $d$. In particular, there exist $(d ; \varepsilon)$-regular graphs which contain relatively large empty bipartite subgraphs (holes). Clearly these holes cannot be too large. The goal of this section is to find the 
maximal size of them. As a corollary, the size of a largest complete bipartite graph that can be contained in a $(d ; \varepsilon)$-regular pair is also given.

Let us begin with some definitions for a bipartite graph $G=\left(V_{1} \cup V_{2}, E\right)$. Set $K(U, W)$ for the complete bipartite graph with vertex sets $U$ and $W$ and define the bipartite complement $\bar{G}=\left(V_{1} \cup V_{2}, K\left(V_{1}, V_{2}\right) \backslash E(G)\right)$ of $G$. The largest integer $r$ such that $K_{r, r} \subseteq G$ is the bipartite clique number $\omega_{\text {bip }}(G)$ of $G$, and the largest integer $r$ such that $K_{r, r} \subseteq \bar{G}$ is the bipartite independence number $\alpha_{\text {bip }}(G)$ of $G$. Clearly, $\alpha_{\text {bip }}(G)=\omega_{\text {bip }}(\bar{G})$. We also set

$$
\begin{aligned}
& \alpha_{\text {bip }}(n ; d, \varepsilon)=\max \left\{\alpha_{\text {bip }}(G): G=\left(V_{1} \cup V_{2}, E\right) \text { is }(d ; \varepsilon) \text {-regular with }\left|V_{1}\right|=\left|V_{2}\right|=n\right\}, \\
& \omega_{\text {bip }}(n ; d, \varepsilon)=\max \left\{\omega_{\text {bip }}(G): G=\left(V_{1} \cup V_{2}, E\right) \text { is }(d ; \varepsilon) \text {-regular with }\left|V_{1}\right|=\left|V_{2}\right|=n\right\} .
\end{aligned}
$$

Our main results determine these parameters asymptotically when $n$ goes to infinity. With given real numbers $d$ and $\varepsilon$ we set $\alpha_{0}=2 \varepsilon(\sqrt{\varepsilon d}-\varepsilon) /(d-\varepsilon)$ and $\omega_{0}=2 \varepsilon(\sqrt{\varepsilon(1-d)}-$ $\varepsilon) /(1-d-\varepsilon)$.

Theorem 2.1 For all real numbers $0<d<1$ there exists $\varepsilon_{0}>0$ such that for all $\varepsilon<\varepsilon_{0}$

$$
\lim _{n \rightarrow \infty} \frac{\alpha_{\text {bip }}(n ; d, \varepsilon)}{n}=\alpha_{0} .
$$

Corollary 2.2 For all real numbers $0<d<1$ there exists $\varepsilon_{0}>0$ such that for all $\varepsilon<\varepsilon_{0}$

$$
\lim _{n \rightarrow \infty} \frac{\omega_{\text {bip }}(n ; d, \varepsilon)}{n}=\omega_{0} .
$$

Proof To prove Corollary 2.2 it is enough to observe that a graph $G$ is $(d ; \varepsilon)$-regular if and only if its bipartite complement $\bar{G}$ is $(1-d, \varepsilon)$-regular.

Remark 2.3 With $\varepsilon \rightarrow 0$ we have $\alpha_{0} \sim 2 \varepsilon^{3 / 2} / \sqrt{d}$ and $\omega_{0} \sim 2 \varepsilon^{3 / 2} / \sqrt{1-d}$.

In fact, one can prove a stronger result than Theorem 2.1. We no longer assume that the bipartition is balanced. Before we make this precise, let us state the formal definition of an $(\alpha, \beta)$-hole.

Definition 2.4 Let $G=\left(V_{1} \cup V_{2}, E\right)$ be a bipartite graph and $0<\alpha, \beta \leq 1$. An $(\alpha, \beta)$ hole is an induced subgraph $(A \cup B, \emptyset)$ of $G$ with $A \subseteq V_{1}, B \subseteq V_{2},|A| \geq \alpha\left|V_{1}\right|$ and $|B| \geq \beta\left|V_{2}\right|$. If $\alpha=\beta$ then we are simply talking about an $\alpha$-hole.

Note that with given $d$ and $\varepsilon$, the size of one set of the bipartition of a largest hole that can be contained in a $(d ; \varepsilon)$-regular pair depends on the size of the other one. Hence, our task is to find the value of the function $\beta_{0}(\alpha ; d, \varepsilon)$ defined as follows:

Definition 2.5 Let $\beta_{0}=\beta_{0}(\alpha ; d, \varepsilon)$ be a real number satisfying the property that for all $\delta>0$ there exists $n_{0}=n_{0}(d, \varepsilon, \alpha, \delta)$ such that:

(a) no $(d ; \varepsilon)$-regular graph $G$ with $\left|V_{1}\right|,\left|V_{2}\right| \geq n_{0}$ contains an $\left(\alpha, \beta_{0}+\delta\right)$-hole,

(b) for all $n \geq n_{0}$ there exists a $(d ; \varepsilon)$-regular graph with $\left|V_{1}\right|=\left|V_{2}\right|=n$ containing an $\left(\alpha, \beta_{0}-\delta\right)$-hole. 
It is easy to see that if the number $\beta_{0}(\alpha ; d, \varepsilon)$ exists then it is unique. Note that for $\varepsilon>d$, the empty graph (a $(1,1)$-hole) is $(d ; \varepsilon)$-regular. One can also show that for $\varepsilon=d$, a $(d ; \varepsilon)$-regular graph may contain any $(\alpha, \beta)$-hole with $\alpha<\varepsilon$ or $\beta<\varepsilon$. Therefore for the rest of the paper we will be assuming that $0<\varepsilon<d<1$.

To prove that a given $\beta_{0}$ is the value of $\beta_{0}(\alpha ; d, \varepsilon)$ at first we show that for all $\beta>\beta_{0}$ there exists $n_{0}$ such that no $(d ; \varepsilon)$-regular graph with at least $n_{0}$ vertices on each side of the bipartition contains an $(\alpha, \beta)$-hole. Then, for any given $\beta<\beta_{0}$ we construct a $(d ; \varepsilon)$ regular graph containing an $(\alpha, \beta)$-hole. In these constructions the densities of some pairs of small subsets can exceed $d+\varepsilon$, but surely can not be larger than one. Therefore for large $d$ and $\varepsilon$ these constructions, and hence the formula of the function $\beta_{0}(\alpha ; d, \varepsilon)$, are different than for small ones.

It turns out that in most cases the value of $\beta_{0}(\alpha ; d, \varepsilon)$ is given by one of the following functions:

$$
f(\alpha)=\frac{2 \varepsilon^{2}(2 \varepsilon-\alpha)}{\alpha(d-\varepsilon)+2 \varepsilon^{2}}, \quad g(\alpha)=\frac{2 \varepsilon^{3}}{\alpha}+\varepsilon(1-d-\varepsilon), \quad h(\alpha)=\frac{2 \varepsilon^{3}}{\alpha-\varepsilon(1-d-\varepsilon)} .
$$

All these functions are decreasing. Note that for $\varepsilon<d$ the equation $\beta=f(\alpha)$ is equivalent to

$$
\left(\beta+\frac{2 \varepsilon^{2}}{d-\varepsilon}\right)\left(\alpha+\frac{2 \varepsilon^{2}}{d-\varepsilon}\right)=\frac{4 \varepsilon^{3} d}{(d-\varepsilon)^{2}} .
$$

Hence the function $f$ is symmetric with respect to the line $\alpha=\beta$, which means that $f=f^{-1}$. Note also, that equations $\beta=g(\alpha)$ and $\beta=h(\alpha)$ are equivalent to

$$
\alpha(\beta-\varepsilon(1-d-\varepsilon))=2 \varepsilon^{3} \text { and }(\alpha-\varepsilon(1-d-\varepsilon)) \beta=2 \varepsilon^{3},
$$

respectively, and therefore $g=h^{-1}$.

Now let us state without the proof results giving the values of the function $\beta_{0}(\alpha ; d, \varepsilon)$. Unfortunately, we do not know this value for $\alpha=2 \varepsilon^{2} /(d+\varepsilon)$. We set $c=c(d, \varepsilon)=$ $(\varepsilon / 2)\left(1-(d+\varepsilon)+\sqrt{1+d^{2}+\varepsilon^{2}+2 \varepsilon d-2 d+6 \varepsilon}\right)$ for the positive solution of the equation $g(\alpha)=h(\alpha)=\alpha$.

Theorem 2.6 For $d \leq 1 / 2$ we have

$$
\beta_{0}(\alpha ; d, \varepsilon)= \begin{cases}1 & \text { for } 0<\alpha<2 \varepsilon^{2} /(d+\varepsilon) \\ f(\alpha) & \text { for } 2 \varepsilon^{2} /(d+\varepsilon)<\alpha<\varepsilon \\ 2 \varepsilon^{2} /(d+\varepsilon) & \text { for } \varepsilon \leq \alpha \leq 1\end{cases}
$$

for $d>1 / 2$ and $\varepsilon<(1-d)^{2} / d<1-d$ we have

$$
\beta_{0}(\alpha ; d, \varepsilon)= \begin{cases}1 & \text { for } 0<\alpha<2 \varepsilon^{2} /(d+\varepsilon), \\ g(\alpha) & \text { for } 2 \varepsilon^{2} /(d+\varepsilon)<\alpha<2 \varepsilon^{2} /(1-d+\varepsilon), \\ f(\alpha) & \text { for } 2 \varepsilon^{2} /(1-d+\varepsilon) \leq \alpha<2 \varepsilon(1-d), \\ h(\alpha) & \text { for } 2 \varepsilon(1-d) \leq \alpha<\varepsilon, \\ 2 \varepsilon^{2} /(d+\varepsilon) & \text { for } \varepsilon \leq \alpha \leq 1,\end{cases}
$$


for $d>1 / 2$ and $(1-d)^{2} / d \leq \varepsilon \leq 1-d$ we have

$$
\beta_{0}(\alpha ; d, \varepsilon)= \begin{cases}1 & \text { for } 0<\alpha<2 \varepsilon^{2} /(d+\varepsilon), \\ g(\alpha) & \text { for } 2 \varepsilon^{2} /(d+\varepsilon)<\alpha<c \\ h(\alpha) & \text { for } c \leq \alpha<\varepsilon \\ 2 \varepsilon^{2} /(d+\varepsilon) & \text { for } \varepsilon \leq \alpha \leq 1\end{cases}
$$

and for $d>1 / 2$ and $\varepsilon>1-d$ we have

$$
\beta_{0}(\alpha ; d, \varepsilon)= \begin{cases}1 & \text { for } 0<\alpha<\varepsilon(1-d+\varepsilon) \\ \left(\varepsilon^{2} / \alpha\right)(1-d+\varepsilon) & \text { for } \varepsilon(1-d+\varepsilon) \leq \alpha<\varepsilon \\ \varepsilon(1-d+\varepsilon) & \text { for } \varepsilon \leq \alpha \leq 1\end{cases}
$$

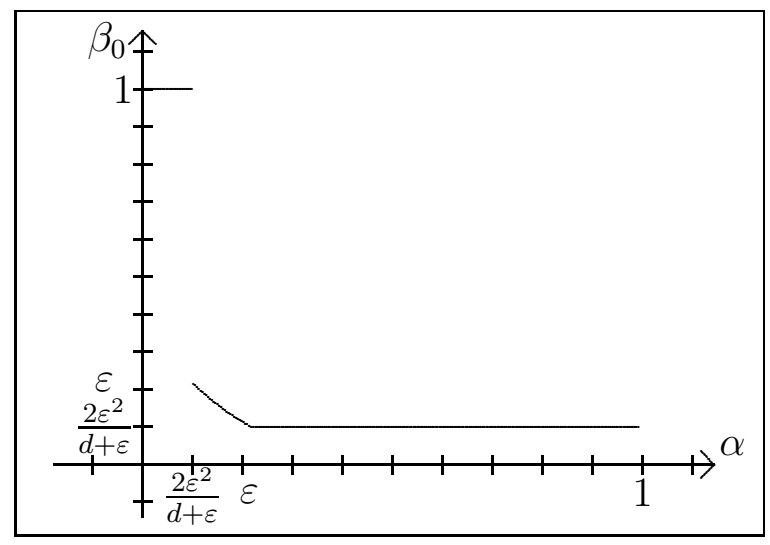

Figure 1: A sketch of the graph of $\beta_{0}=\beta_{0}(\alpha ; d, \varepsilon)$ as a function of $\alpha$ for $d=0.5$ and $\varepsilon=0.2$

Note that since a bipartite graph is $(d ; \varepsilon)$-regular if and only if its bipartite complement is $(1-d ; \varepsilon)$-regular, we can simply replace $d$ by $1-d$ in the above results to get the size of a largest complete bipartite subgraph that can be contained in a $(d ; \varepsilon)$-regular graph.

\section{The Proof of Theorem 2.1}

Before we prove Theorem 2.1, we state a result showing how, by using random graphs, one can find a $(d ; \varepsilon)$-regular bipartite graph for any given real numbers $d, \varepsilon \in(0,1)$. For a later application, we give it here in a more general form.

Fact 3.1 For all real numbers $d, \varepsilon \in(0,1)$ and $\gamma>0$, there exists $n_{0}=n_{0}(d, \varepsilon, \gamma)$ such that for all $n \geq n_{0}$, there exists a $(d ; \varepsilon)$-regular bipartite graph $G=\left(V_{1} \cup V_{2}, E\right)$ with $\left|V_{1}\right|=n$ and $\left|V_{2}\right|=\lceil\gamma n\rceil$. 
Proof Without loss of generality we may assume that $\gamma n$ is integer. Let $G=G(n, \gamma n, d)$ $=\left(V_{1} \cup V_{2}, E\right)$ be a random bipartite graph with $\left|V_{1}\right|=n,\left|V_{2}\right|=\gamma n$, and edge probability d. Moreover, for each pair of subsets $U, W, U \subset V_{1}, W \subset V_{2},|U|=\varepsilon n,|W|=\varepsilon \gamma n$, let

$$
X_{U, W}=\mathrm{e}_{G}(U, W)
$$

denote a random variable counting edges between sets $U$ and $W$. Note, that each of these random variables has the same binomial distribution with expected value $\mu=|U||W| d=$ $\varepsilon^{2} \gamma n^{2} d$. Applying Chernoff's inequality (see inequality (2.9) in [1]) with $\epsilon=n^{-\frac{1}{3}}$ we get

$$
\begin{aligned}
& \mathbb{P}\left(\exists U, W:\left|X_{U, W}-\mu\right| \geq n^{-\frac{1}{3}} \mu\right) \leq 2^{n} 2^{\gamma n} \mathbb{P}\left(|X-\mu| \geq n^{-\frac{1}{3}} \mu\right) \\
\leq & \left(2^{1+\gamma}\right)^{n} 2 \exp \left\{-\frac{n^{-\frac{2}{3}}}{3} \mu\right\}=\left(2^{1+\gamma}\right)^{n} 2 \exp \left\{-\frac{\varepsilon^{2} \gamma n^{\frac{4}{3}} d}{3}\right\}=o(1),
\end{aligned}
$$

where $X$ has the same distribution as all random variables $X_{U, W}$. Therefore there exists a graph $G$ with vertex set $V_{1} \cup V_{2}$ such that for each pair of subsets $U, W$ like above we have

$$
\left|\mathrm{d}_{G}(U, W)-d\right|<\frac{d}{n^{\frac{1}{3}}}=\varepsilon_{0},
$$

thus $G$ is $\left(d ; \varepsilon, \varepsilon_{0}\right)$-regular.

Now we are ready to prove Theorem 2.1.

\section{Proof of Theorem 2.1}

To prove Theorem 2.1 we have to show that

$$
\underset{0<d<1}{\forall} \quad \underset{\varepsilon_{0}>0}{\exists} \quad \underset{\varepsilon<\varepsilon_{0}}{\forall} \quad \underset{\delta>0}{\forall} \quad \underset{N \in \mathbb{N}}{\exists} \quad \underset{n>N}{\forall}
$$

the following to statements are true:

(i) There exists a $(d ; \varepsilon)$-regular bipartite graph $G=\left(V_{1} \cup V_{2}, E\right),\left|V_{1}\right|=\left|V_{2}\right|=n$, containing a $(2 \varepsilon(\sqrt{\varepsilon d}-\varepsilon) /(d-\varepsilon)-\delta)$-hole.

(ii) No $(d ; \varepsilon)$-regular graph $G=\left(V_{1} \cup V_{2}, E\right),\left|V_{1}\right|=\left|V_{2}\right|=n$, contains a $(2 \varepsilon(\sqrt{\varepsilon d}-\varepsilon) /(d-$ $\varepsilon)+\delta$ ) -hole.

We start with the proof of the part (i). For any $0<d<1$ let

$$
\varepsilon_{0}=\min \left\{\frac{(1-d)^{2}}{d}, 1-d, d\right\} .
$$

Further for any $\varepsilon<\varepsilon_{0}$ and $\delta>0$ let $N \in \mathbb{N}$ be as large as needed. Now for any $n>N$ we will construct a $(d ; \varepsilon)$-regular graph $G=\left(V_{1} \cup V_{2}, E\right),\left|V_{1}\right|=\left|V_{2}\right|=n$, containing a $(2 \varepsilon(\sqrt{\varepsilon d}-\varepsilon) /(d-\varepsilon)-\delta)-$ hole.

Let

$$
\alpha=\frac{2 \varepsilon(\sqrt{\varepsilon d}-\varepsilon)}{d-\varepsilon}-\delta \quad \text { and } \quad \alpha^{\prime}=\frac{2 \varepsilon(\sqrt{\varepsilon d}-\varepsilon)}{d-\varepsilon} .
$$


Next we define

$$
\begin{gathered}
\xi=\frac{1}{3} \varepsilon^{2}\left(1-\frac{\alpha}{\alpha^{\prime}}\right)^{2} \alpha, \\
\xi^{\prime}=\frac{1}{8} \varepsilon(\varepsilon-\alpha) \xi \\
d_{1}=d-\varepsilon+2 \xi \quad \text { and } \quad d_{2}=d_{3}=d-\varepsilon+2 \frac{\varepsilon^{2}}{\alpha^{\prime}}-\xi .
\end{gathered}
$$

Note that $\alpha^{\prime}>2 \varepsilon^{2} /(1-d+\varepsilon)$ and therefore $d_{2}=d_{3}<1-\xi$. We construct the desired graph as follows. We take four disjoint sets of vertices $A, B,|A|=|B|=\lceil\alpha n\rceil, V_{1}$ and $V_{2},\left|V_{1}\right|=\left|V_{2}\right|=n-\lceil\alpha n\rceil$ and three graphs

$$
G_{1}=\left(V_{1} \cup V_{2}, E\left(V_{1}, V_{2}\right)\right), \quad G_{2}=\left(B \cup V_{1}, E\left(B, V_{1}\right)\right), \quad G_{3}=\left(A \cup V_{2}, E\left(A, V_{2}\right)\right),
$$

where $G_{i}$ is $\left(d_{i} ; \xi^{\prime}, \xi\right)$-regular, $i=1,2,3$, guaranteed by Fact 3.1 . We set

$$
G=G_{1} \cup G_{2} \cup G_{3}=\left(\left(A \cup V_{1}\right) \cup\left(B \cup V_{2}\right), E\left(V_{1}, V_{2}\right) \cup E\left(B, V_{1}\right) \cup E\left(A, V_{2}\right)\right) .
$$

By the construction, $G$ contains a $(2 \varepsilon(\sqrt{\varepsilon d}-\varepsilon) /(d-\varepsilon)-\delta)$-hole, to complete the proof it remains to show that $G$ is $(d ; \varepsilon)$-regular. To prove this, let $U \subset A \cup V_{1}, W \subset B \cup V_{2}$, be any subsets of the set of vertices, $|U|=|W|=\lceil\varepsilon n\rceil$. We set $A^{\prime}=A \cap U, B^{\prime}=B \cap W$, $U^{\prime}=U \cap V_{1}, W^{\prime}=W \cap V_{2}$, and let $\left|A^{\prime}\right|=a n \leq\lceil\alpha n\rceil<\alpha^{\prime} n,\left|B^{\prime}\right|=b n \leq\lceil\alpha n\rceil<\alpha^{\prime} n$ (see Figure 2).

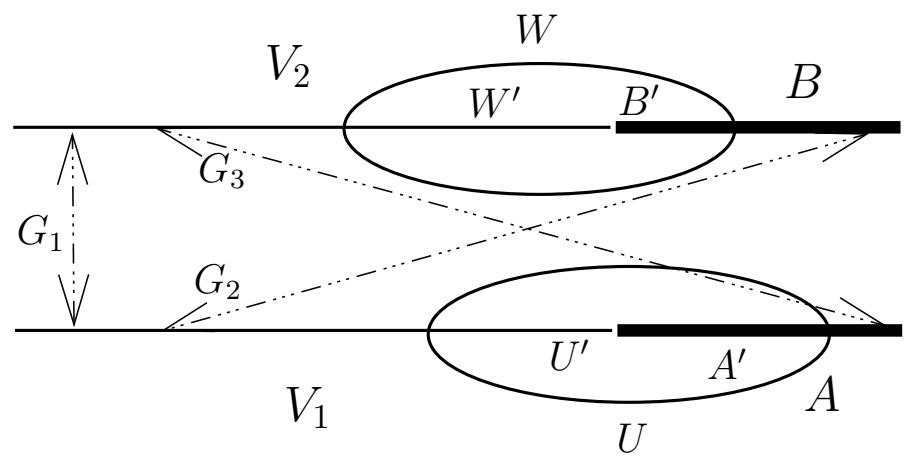

Figure 2: The construction of the graph $G$ and the sets $U$ and $W$

Then we get

$$
\begin{aligned}
& \mathrm{d}_{G}(U, W)=\frac{a(\varepsilon-b)}{\varepsilon^{2}} \mathrm{~d}_{G_{3}}\left(A^{\prime}, W^{\prime}\right)+\frac{b(\varepsilon-a)}{\varepsilon^{2}} \mathrm{~d}_{G_{2}}\left(B^{\prime}, U^{\prime}\right) \\
& +\frac{(\varepsilon-a)(\varepsilon-b)}{\varepsilon^{2}} \mathrm{~d}_{G_{1}}\left(U^{\prime}, W^{\prime}\right)+O\left(\frac{1}{n}\right) .
\end{aligned}
$$


Note that for any choice of $U$ and $W$, by (3), we have $\left|U^{\prime}\right|>\xi^{\prime} n,\left|W^{\prime}\right|>\xi^{\prime} n$, and thus we may use the $\left(d_{1} ; \xi^{\prime}, \xi\right)$-regularity of $G_{1}$ to bound the density $\mathrm{d}_{G_{1}}\left(U^{\prime}, W^{\prime}\right)$ as follows:

$$
d-\varepsilon+\xi<\mathrm{d}_{G_{1}}\left(U^{\prime}, W^{\prime}\right)<d-\varepsilon+3 \xi .
$$

Unfortunately, both sets, $A^{\prime}$ and $B^{\prime}$, can be smaller then $\xi^{\prime} n$. In these cases we will be assuming that $0 \leq \mathrm{d}_{G_{3}}\left(A^{\prime}, W^{\prime}\right) \leq 1$ and $0 \leq \mathrm{d}_{G_{2}}\left(B^{\prime}, U^{\prime}\right) \leq 1$ respectively. Otherwise, we will use the $\left(d_{i} ; \xi^{\prime}, \xi\right)$-regularity of the graphs $G_{i}, i=2,3$, to get

$$
\begin{aligned}
& d-\varepsilon+2 \frac{\varepsilon^{2}}{\alpha^{\prime}}-2 \xi<\mathrm{d}_{G_{2}}\left(B^{\prime}, U^{\prime}\right)<d-\varepsilon+2 \frac{\varepsilon^{2}}{\alpha^{\prime}}, \\
& d-\varepsilon+2 \frac{\varepsilon^{2}}{\alpha^{\prime}}-2 \xi<\mathrm{d}_{G_{3}}\left(A^{\prime}, W^{\prime}\right)<d-\varepsilon+2 \frac{\varepsilon^{2}}{\alpha^{\prime}} .
\end{aligned}
$$

Therefore, in order to prove that $d-\varepsilon<\mathrm{d}_{G}(U, W)<d+\varepsilon$, by (4), (5), (6) and (7), we have to show the validity of the following inequalities:

$$
\begin{gathered}
\frac{a(\varepsilon-b)}{\varepsilon^{2}}+\frac{b(\varepsilon-a)}{\varepsilon^{2}}+\frac{(\varepsilon-a)(\varepsilon-b)}{\varepsilon^{2}}(d-\varepsilon+3 \xi)+O\left(\frac{1}{n}\right)<d+\varepsilon, \\
\frac{(\varepsilon-a)(\varepsilon-b)}{\varepsilon^{2}}(d-\varepsilon+\xi)+O\left(\frac{1}{n}\right)>d-\varepsilon,
\end{gathered}
$$

where $\left|A^{\prime}\right|<\xi^{\prime} n$ and $\left|B^{\prime}\right|<\xi^{\prime} n$. This follows from (2) and (3).

In the case, when $\left|A^{\prime}\right|<\xi^{\prime} n,\left|B^{\prime}\right| \geq \xi^{\prime} n$ (or similarly, when $\left|A^{\prime}\right| \geq \xi^{\prime} n,\left|B^{\prime}\right|<\xi^{\prime} n$ ) we get

$$
\begin{gathered}
\frac{a(\varepsilon-b)}{\varepsilon^{2}}+\frac{b(\varepsilon-a)}{\varepsilon^{2}}\left(d-\varepsilon+2 \frac{\varepsilon^{2}}{\alpha^{\prime}}\right)+\frac{(\varepsilon-a)(\varepsilon-b)}{\varepsilon^{2}}(d-\varepsilon+3 \xi)+O\left(\frac{1}{n}\right)<d+\varepsilon, \\
\frac{b(\varepsilon-a)}{\varepsilon^{2}}\left(d-\varepsilon+2 \frac{\varepsilon^{2}}{\alpha^{\prime}}-2 \xi\right)+\frac{(\varepsilon-a)(\varepsilon-b)}{\varepsilon^{2}}(d-\varepsilon+\xi)+O\left(\frac{1}{n}\right)>d-\varepsilon .
\end{gathered}
$$

Here, to prove the last inequality, apart from (2) and (3), we use also the fact that $\varepsilon \leq 1 / 2$.

Finally, if $\left|A^{\prime}\right| \geq \xi^{\prime} n$ and $\left|B^{\prime}\right| \geq \xi^{\prime} n$, by (2), we have

$$
\begin{aligned}
& f_{1}(a, b)=\frac{a(\varepsilon-b)}{\varepsilon^{2}}\left(d-\varepsilon+2 \frac{\varepsilon^{2}}{\alpha^{\prime}}\right)+\frac{b(\varepsilon-a)}{\varepsilon^{2}}\left(d-\varepsilon+2 \frac{\varepsilon^{2}}{\alpha^{\prime}}\right)+ \\
& \frac{(\varepsilon-a)(\varepsilon-b)}{\varepsilon^{2}}(d-\varepsilon+3 \xi)+O\left(\frac{1}{n}\right)< \\
& d-\varepsilon+2 \varepsilon\left(\frac{b}{\alpha^{\prime}}-2 \frac{a b}{\alpha^{\prime 2}}+\frac{a}{\alpha^{\prime}}\right)+3 \xi<d+\varepsilon,
\end{aligned}
$$




$$
\begin{aligned}
& f_{2}(a, b)=\frac{a(\varepsilon-b)}{\varepsilon^{2}}\left(d-\varepsilon+2 \frac{\varepsilon^{2}}{\alpha^{\prime}}-2 \xi\right)+\frac{b(\varepsilon-a)}{\varepsilon^{2}}\left(d-\varepsilon+2 \frac{\varepsilon^{2}}{\alpha^{\prime}}-2 \xi\right)+ \\
& \frac{(\varepsilon-a)(\varepsilon-b)}{\varepsilon^{2}}(d-\varepsilon+\xi)+O\left(\frac{1}{n}\right)= \\
& d-\varepsilon+2 \varepsilon\left(\frac{b}{\alpha^{\prime}}-2 \frac{a b}{\alpha^{\prime 2}}+\frac{a}{\alpha^{\prime}}\right)+\xi \frac{\varepsilon^{2}-3 \varepsilon a-3 \varepsilon b+5 a b}{\varepsilon^{2}}+O\left(\frac{1}{n}\right)>d-\varepsilon .
\end{aligned}
$$

Since both, $f_{1}(a, b)$ and $f_{2}(a, b)$, are double linear functions, they achieve their extreme values in the corners of the rectangle, on which they are defined. Therefore, to finish the proof of the part (i) of Theorem 2.1, we need to check the validity of the last inequality only at points $(a, b)$ equal to $(0,0),(0, \alpha+1 / n),(\alpha+1 / n, 0)$ and $(\alpha+1 / n, \alpha+1 / n)$.

Now we can move to part (ii). For any real number $d \in(0,1)$ we set $\varepsilon_{0}=\min \{d, 1-d\}$. Now, for any $\varepsilon<\varepsilon_{0}$ and $\delta>0$ we define

$$
N=\left\lceil\frac{2(\sqrt{\varepsilon d}-\varepsilon)}{\delta(d-\varepsilon)}\right\rceil \text {. }
$$

Take any $n>N$ and let $G=\left(V_{1} \cup V_{2}, E\right),\left|V_{1}\right|=\left|V_{2}\right|=n$, be a $(d ; \varepsilon)$-regular bipartite graph. Suppose, for a contradiction, that $G$ contains a $(2 \varepsilon(\sqrt{\varepsilon d}-\varepsilon) /(d-\varepsilon)+\delta)$-hole between sets $A \subset V_{1}$ and $B \subset V_{2}$.

Without loss of the generality we may assume, that

$$
|A|=|B|=\left\lceil\left(\frac{2 \varepsilon(\sqrt{\varepsilon d}-\varepsilon)}{d-\varepsilon}+\delta\right) n\right\rceil=r
$$

and also that $r<\lceil\varepsilon n\rceil$, since otherwise we would get a contradiction with the $(d ; \varepsilon)$ regularity of $G$. Note also that since $n>N$, we have

$$
\frac{r}{\lceil\varepsilon n\rceil}>\frac{2(\sqrt{\varepsilon d}-\varepsilon)}{d-\varepsilon} \text {. }
$$

We take two sets $U \subset V_{1} \backslash A,|U|=\lceil\varepsilon n\rceil-r$, and $W \subset V_{2} \backslash B,|W|=\lceil\varepsilon n\rceil$ in such a way that $\mathrm{d}_{G}(U, W)>d-\varepsilon$. Since $\left|V_{1} \backslash A\right|>\varepsilon n$, we have $\mathrm{d}_{G}\left(V_{1} \backslash A, W\right)>d-\varepsilon$, and therefore, by Fact 1.1 , this choice is possible. Note that by the $(d ; \varepsilon)$-regularity of $G$ we get $\mathrm{d}_{G}(A \cup U, W)<d+\varepsilon$ and thus

$$
\begin{aligned}
& (d-\varepsilon)|U||W|+\mathrm{d}_{G}(A, W)|A||W|<\mathrm{e}_{G}(U, W)+\mathrm{e}_{G}(A, W)= \\
& \mathrm{e}_{G}(A \cup U, W)<(d+\varepsilon)\lceil\varepsilon n\rceil|W| .
\end{aligned}
$$

Hence

$$
\mathrm{d}_{G}(A, W)<\frac{(d+\varepsilon)\lceil\varepsilon n\rceil-(d-\varepsilon)(\lceil\varepsilon n\rceil-|A|)}{|A|}=d-\varepsilon+\frac{2 \varepsilon\lceil\varepsilon n\rceil}{r}
$$


Therefore, by Fact 1.1, we may choose a set $W^{\prime} \subset W,\left|W^{\prime}\right|=\lceil\varepsilon n\rceil-r$, in such a way that $\mathrm{d}_{G}\left(A, W^{\prime}\right)<d-\varepsilon+2 \varepsilon\lceil\varepsilon n\rceil / r$. Next we take $U^{\prime} \subset V_{1} \backslash A,\left|U^{\prime}\right|=\lceil\varepsilon n\rceil-r$, with $\mathrm{d}_{G}\left(U^{\prime}, B \cup W^{\prime}\right)<d+\varepsilon$. We will show that $d_{G}\left(A \cup U^{\prime}, B \cup W^{\prime}\right)<d-\varepsilon$ getting a contradiction with the $(d ; \varepsilon)$-regularity of $G$. Indeed, we have

$$
\begin{aligned}
& \mathrm{d}_{G}\left(A \cup U^{\prime}, B \cup W^{\prime}\right)=\frac{\mathrm{d}_{G}\left(U^{\prime}, B \cup W^{\prime}\right)\left|U^{\prime}\right|\lceil\varepsilon n\rceil}{\lceil\varepsilon n\rceil^{2}}+\frac{\mathrm{d}_{G}\left(A, W^{\prime}\right)|A|\left|W^{\prime}\right|}{\lceil\varepsilon n\rceil^{2}}< \\
& (d+\varepsilon)\left(1-\frac{r}{\lceil\varepsilon n\rceil}\right)+\left(d-\varepsilon+\frac{2 \varepsilon\lceil\varepsilon n\rceil}{r}\right) \frac{r}{\lceil\varepsilon n\rceil}\left(1-\frac{r}{\lceil\varepsilon n\rceil}\right)= \\
& d+3 \varepsilon-4 \varepsilon \frac{r}{\lceil\varepsilon n\rceil}-(d-\varepsilon)\left(\frac{r}{\lceil\varepsilon n\rceil}\right)^{2}< \\
& d+3 \varepsilon-4 \varepsilon \frac{2(\sqrt{\varepsilon d}-\varepsilon)}{d-\varepsilon}-(d-\varepsilon)\left(\frac{2(\sqrt{\varepsilon d}-\varepsilon)}{d-\varepsilon}\right)^{2}=d-\varepsilon .
\end{aligned}
$$

\section{Applications}

In this section we present the degree condition of vertices in $(d ; \varepsilon)$-regular graphs that guarantees their connection by a path. More studies about this problem can be found in [5]. By $\operatorname{dist}_{G}(x, y)$ we denote the distance of vertices $x, y \in V$, that is, the length of a shortest path connecting them, if such a path exists. Otherwise we set $\operatorname{dist}_{G}(x, y)=\infty$. By the diameter of $G$ we mean $\operatorname{diam}(G)=\max _{x, y \in V} \operatorname{dist}_{G}(x, y)$. In particular, if $G$ is not connected, then $\operatorname{diam}(G)=\infty$.

Theorem 4.1 In any $(d ; \varepsilon)$-regular bipartite graph $G$, where $0<\varepsilon \leq d \leq 1-\varepsilon$, if $\operatorname{deg}_{G}(v), \operatorname{deg}_{G}(w)>2 \varepsilon^{2} n /(d+\varepsilon)$, then

$$
\operatorname{dist}_{G}(v, w) \leq \begin{cases}5 & \text { if } v \in V_{i}, w \in V_{j} \\ 4 & \text { if } v, w \in V_{i} .\end{cases}
$$

Proof Let $0<\varepsilon \leq d \leq 1-\varepsilon$ and let a $(d ; \varepsilon)$-regular bipartite graph $G$ be given. Furthermore let $v, w \in V$, $\operatorname{deg}_{G}(v)>2 \varepsilon^{2} n /(d+\varepsilon), \operatorname{deg}_{G}(w)>2 \varepsilon^{2} n /(d+\varepsilon)$. We set $A=N_{G}(v), B=N_{G}(w)$. Without loss of generality, we may assume that $v \in V_{1}$. As the first one we will consider the case where $w \in V_{1}$. We let $C \subseteq V_{1}$ be the set of all vertices adjacent to some vertex of $B$. Then $|C| \geq n-\varepsilon n \geq \varepsilon n$, since otherwise the sets $B$ and $V_{1} \backslash C$ would provide an $(\alpha, \varepsilon)$-hole, where $\alpha>2 \varepsilon^{2} /(d+\varepsilon)$, which contradicts Theorem 2.6. Therefore $\mathrm{e}_{G}(A, C)>0$ and so the vertices $v$ and $w$ are connected in $G$ by a path of length at most 4.

Now we turn to the situation where $w \in V_{2}$. Similarly to the above, the set of vertices $C \subseteq V_{2}$ adjacent to some vertex of $B$ has cardinality $|C| \geq n-\varepsilon n \geq \varepsilon n$. Now we repeat the reasoning from the first part of the proof to the sets $A$ and $C$, getting a path of length at most 5 (see Figure 3). 


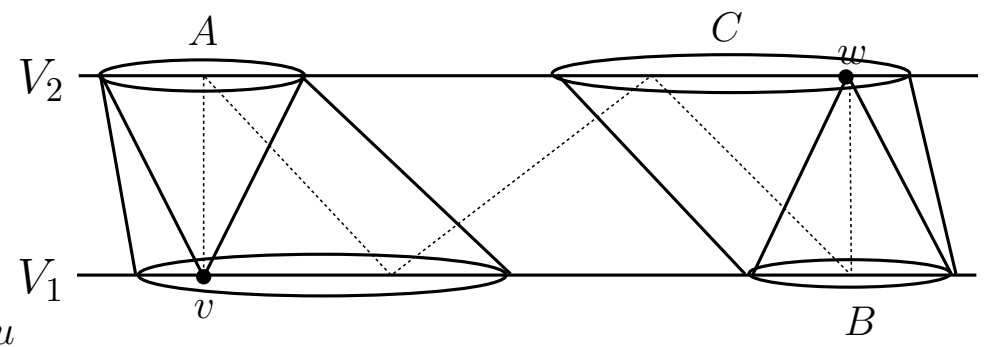

Figure 3: The path from $v$ to $w$, where $w \in V_{2}$

Corollary 4.2 For each $(d ; \varepsilon)$-regular bipartite graph $G$, where $0<\varepsilon \leq d \leq 1-\varepsilon$, if $\delta_{G}>2 \varepsilon^{2} n /(d+\varepsilon)$, then $\operatorname{diam}(G) \leq 5$.

It turns out that the above result is the best possible, namely that there exist $(d, \varepsilon)$ regular graphs containing a vertex with degree slightly smaller then $2 \varepsilon^{2} n /(d+\varepsilon)$, which is not connected by a path with any other vertices except of its neighbors.

Theorem 4.3 For all real numbers $\varepsilon, d, \alpha \in(0,1)$ where $0<\varepsilon \leq d \leq 1-\varepsilon$ and $\alpha<$ $2 \varepsilon^{2} n /(d+\varepsilon)$, there exists a $(d ; \varepsilon)$-regular graph $G$ containing an isolated star with an edges, and therefore there exists a vertex of degree $\alpha n$, which is connected (by a path) only with its neighbors.

Sketch of the proof According to Theorem 2.6, there exists a $(d ; \varepsilon)$-regular graph $G$ containing an $(\alpha, 1)$-hole spanned between sets $A \subset V_{1}$ and $V_{2}$. We add to $V_{2}$ one vertex $w$ and connect it with all vertices of $A$. This addition has a very small impact on the regularity of $G$ (for details see [5]). So we have gotten an isolated star with $\alpha n$ edges, as required (see Figure 4).

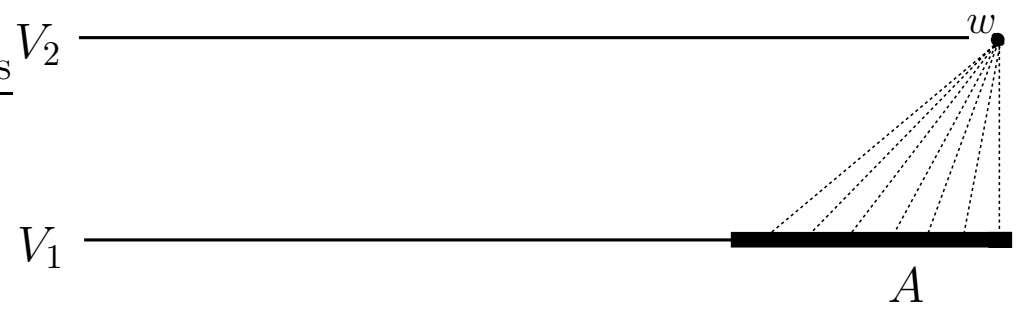

Figure 4: The graph $G$ with a new vertex $w$

\section{Acknowledgements}

I would like to thank Andrzej Ruciński for suggesting this problem and for his help and advice in preparing this manuscript. I also would like to thank Referee for his valuable comments on the presentation of this paper. 


\section{References}

[1] S. Janson, T. Łuczak \& A. Ruciński, Random Graphs, John Wiley and Sons, New York 2000.

[2] J. Komlós, G.N. Sárközy \& E. Szemerédi, On the square of a Hamiltonian cycle in dense graphs, Random Structures Algorithms 9 (1996), no. 1-2, 193-211.

[3] J. Komlós, G.N. Sárközy \& E. Szemerédi, On the Pósa-Seymour conjecture, J. Graph Theory 29 (1998), 167-176.

[4] Y. Peng, V. Rödl \& A. Ruciński, Holes in graphs, Electron. J. Combin. 8 (2001), \#R13.

[5] J. Polcyn \& A. Ruciński, Short paths in E-regular pairs and small diameter decompositions of dense graphs, submitted.

[6] E. Szemerédi, Regular partitions of graphs, Problèmes combinatoires et théorie des graphes (Colloq. Internat. CNRS, Univ. Orsay, Orsay, 1976) (Bermond, J.-C., Fournier, J.-C., Las Vergnas, M., Sotteau, D., eds),(1976), pp. 399-401. 